\title{
REJECTION REACTIONS TO DIFFERENT ORGAN TRANSPLANTS
}

\author{
P. J. FRIEND \\ Cambridge
}

Rejection of solid organ grafts is conventionally classified as hyperacute, acute and chronic. Hyperacute rejection is the consequence of preformed antibodies in the serum of the recipient which recognise donor antigens. This causes rapid activation of complement, platelet aggregation, thrombosis and ischaemic necrosis. The graft is destroyed very rapidly (within minutes or hours). The patients at risk of hyperacute rejection are those who have previously been exposed to antigenic stimuli from multiple sources, particularly patients who have received multiple transfusions, had multiple pregnancies or previous transplants. In order to prevent this occurrence, patients who are known to be at risk are screened for lymphocytotoxic antibodies against a potential donor at the time the donor becomes available.

Hyperacute rejection is very rare since the introduction of cross-match techniques in order to prevent transplantation of a kidney into a sensitised donor. This phenomenon has also been recognised in other forms of solid organ transplantation. Although it has been recognised following liver transplantation, its incidence is exceedingly rare. ${ }^{1}$

Acute rejection usually occurs early following transplantation (typically within 4 weeks). It is a classical cell-mediated immune response involving presentation of foreign antigens to $T$ cells by antigen presenting cells, proliferation and activation of $\mathrm{T}$ cell clones and destruction of the graft by cytoxic $\mathrm{T}$ cells. Acute rejection is very common following organ transplantation $(50-70 \%)$ and usually responds to increased immunosuppression.

Chronic rejection occurs later (typically months or years after transplantation). It is manifest by a progressive arteriopathy leading to ischaemic changes. There are also other lesions which are specific to the individual organ transplants. In renal

Correspondence to: P. J. Friend, MA, MD, FRCS, Department of Surgery, Level 9, Addenbrooke's Hospital, Hills Road, Cambridge CB2 2QQ. transplantation tubular atrophy and glomerulosclerosis are associated with chronic rejection. In heart transplantation the phenomenon of 'accelerated atherosclerosis' is recognised as is 'obliterative bronchiolitis' following lung transplantation. The syndrome of 'vanishing bile duct syndrome' following liver transplantation is diagnostic of chronic rejection. These histological appearances do not appear to be related to cellular infiltration of the graft and are assumed to be humoral in aetiology. Chronic rejection is very largely unresponsive to alteration in the immunosuppressive protocol and remains a major source of graft loss in all areas of solid organ transplantation.

The objective of immunosuppression is to inactivate or remove immune effector cells in order to prevent rejection without placing the patient at undue risk of infection or tumour. The drugs which are used for maintenance immunosuppression include some or all of the long-established agents cyclosporin, azathioprine and prednisolone. Episodes of acute rejection are treated either with a high dose of steroid or injections of antibodies which target human lymphocytes; these may be polyclonal (antilymphocyte globulin, anti-thymocyte globulin) or monoclonal.

The limitations of current immunosuppression include the lack of specificity of the current drugs; this leads to a risk of infection and a long-term risk of tumour development ${ }^{2}$ (possibly as a result of the suppression of immune surveillance ${ }^{3}$ ).

A second major problem of current therapy is that of toxicity. The drugs currently in use all have toxic effects which limit the dose at which they can be used. The strategy for immunosuppressive therapy in most transplant units includes the use of several drugs at a lower dose in order to reduce the toxicity of each drug. ${ }^{4}$

The level of immunosuppression which is desirable depends upon the organ which has been transplanted. For example, heart transplants generally 
require a higher dose of immunosuppression than liver transplants. In terms of immunosuppression, the risks of rejection are balanced against the risk of treatment. Clearly for an organ which is not absolutely essential for life (for example a kidney) it would not be acceptable to place the patient at a very considerable risk of death from infection in order to prevent the rejection of the graft. On the other hand, for a life-preserving organ (heart or liver) a clinician will be prepared to run greater risks of the complications of the drugs rather than risk losing the graft through rejection.

The immunological properties of the transplanted liver have been the subject of considerable interest for many years. It was first noted following experimental pig liver transplantation that other organs from the same donor appear to benefit from donorspecific protection. ${ }^{5}$ The same effect was demonstrated in the rat and a protective factor identified within the serum. ${ }^{6}$ It has been suggested that this factor is soluble MHC class I antigen. The clinical relevance of this has been demonstrated by the relative protection of kidney ${ }^{7}$ and small bowel ${ }^{8}$ allografts when transplanted in conjunction with a liver from the same donor. It has been shown that, following liver transplantation, donor HLA class I antigen appears within the circulation very rapidly following reperfusion of the graft. ${ }^{9}$

This phenomenon may also explain the relative immunity of the liver from hyperacute rejection and the apparent absence of the long-term graft attrition which is a feature of heart and kidney transplantation. Despite this, however, acute rejection occurs as frequently following liver transplantation as it does with other organ grafts.

Recently the phenomenon of migration of donor leucocytes from the graft into the recipient has been an area of great interest. Particularly following liver transplantation, this leads to a state of 'microchimerism'; whether this phenomenon is related to longterm graft acceptance is not yet agreed. ${ }^{10}$

Despite advances in the knowledge of transplanta- tion immunology and the developments in immunosuppressive drugs over the last 35 years, rejection remains the major cause of graft loss. Chronic rejection remains a very major problem following transplantation of all solid organs and the mechanism of this remains unknown. Current clinical immunosuppression remains non-specific and has a high associated morbidity and mortality. There is considerable interest in the mechanisms of inducing tolerance, or donor-specific hyporesponsiveness, and it is in these areas that one may expect the greatest developments in the future.

\section{REFERENCES}

1. Bird G, Friend, PJ, Donaldson P, O'Grady J, Portmann B, Calne R, Williams R. Hyperacute rejection of the liver. Transplant Proc 1989;21:3742-4.

2. Penn I, Hammond W, Brettschneider L, Starzl TE. Malignant lymphomas in transplant patients. Transplant Proc 1969;1:106-12.

3. Keast D. Immunosurveillance and cancer. Lancet 1970;2:710-2.

4. Opelz G. Comparison of immunosuppressive protocols in renal transplantation: a multicentre view. Transplant Proc 1988;20:31-6.

5. Calne RY, Sells RA, Pena JR, Davis DR, Millar PR, Herbertson BM, et al. Induction of immunological tolerance by porcine liver allografts. Nature 1969;223:472-6.

6. Kamada N. Fully allogeneic liver grafting and the induction of donor-specific unreactivity. Transplantation Proc 1982;13:837-41.

7. Rasmussen A, Davies HFFS, Jamieson NV, Evans DB, Calne RY. Combined transplantation of liver and kidney from the same donor protects the kidney from rejection and improves kidney graft survival. Transplantation 1995;(in press).

8. Grant DR, Wall W, Mimeault R, Zhong R, Ghent C, Garcia B, et al. Successful small-bowel/liver transplantation. Lancet 1990;335:181-4.

9. Pollard SG, Davies HFFS, Calne RY. Clearance of liver graft derived soluble class I antigen following retransplantation. Transplantation 1994;57:856-8.

10. Demetris AJ, Murase N, Starzl TE. Donor dendritic cells after liver and heart allotransplantation under short-term immunosuppression. Lancet 1992;339:1610. 